\title{
Characterization of microaneurysm closure after focal laser photocoagulation in diabetic macular edema
}

\author{
Su Na Lee, Jay Chhablani, Candy K. Chan, Haiyan Wang, Giulio Barteselli, Sharif El-Emam, \\ Maria L. Gomez, Igor Kozak, Lingyun Cheng, and William R. Freeman \\ Department of Ophthalmology, Jacobs Retina Center at Shiley Eye Center, University of \\ California San Diego, La Jolla CA
}

\begin{abstract}
Purpose-To characterize microaneurysm closure following focal laser photocoagulation in diabetic macular edema (DME) using simultaneous fluorescein angiography (FA) and spectraldomain optical coherence tomography (SD-OCT)

Design-Retrospective observational case series.

Methods-Leaking microaneurysms ( $\mathrm{N}=123)$ were analyzed in eyes $(\mathrm{N}=29)$ with nonproliferative diabetic retinopathy (NPDR) which underwent navigated focal laser photocoagulation in DME and followed at 3, 6, and 12 months. Closure of diabetic microaneurysms was characterized in detail following focal laser using SD-OCT.
\end{abstract}

Results-Closure rate of microaneurysms by both FA and SD-OCT was 69.9\% (84/123), $79.7 \%$ (98/123) and $82.9 \%(102 / 123)$ at 3, 6, and 12 months, respectively. Microaneurysm closure rate increased at 6 and 12 months compared to 3 months $(P<.003, P<.001)$. Over half of closed microaneurysms (45/86, $52.3 \%)$ left hyper-reflective spots while remaining half $(41 / 86,47.7 \%)$ disappeared without any hyper-reflectivity by SD-OCT at 3 months. Hyper-reflective spots decreased at $6(36 / 99,36.4 \%)$ and 12 months $(17 / 102,16.7 \%)$ with a concomitant increase in complete loss of reflectivity at $6(63 / 99,63.6 \%)$ and 12 months $(85 / 102,83.3 \%)$. Smaller outer and inner diameters, and heterogeneous lumen reflectivity were positively associated with microaneurysm closure at 12 months $(P<.0001, P<.001, P<.03)$.

Conclusions-Characterization of microaneurysms following focal laser photocoagulation resulted in hyper-reflective spots and complete resolution of all reflectivity using SD-OCT. Smaller microaneurysms and those with heterogeneous lumen were positively associated with microaneurysm closure. These findings provide greater understanding of localized retinal changes following focal laser photocoagulation in DME treatment.

\footnotetext{
(C) 2012 Elsevier Inc. All rights reserved

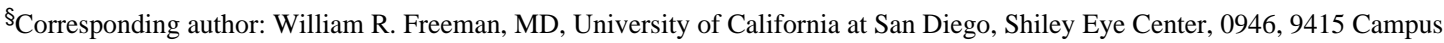
Point Drive, La Jolla, CA 92037; freeman@eyecenter.ucsd.edu.

Publisher's Disclaimer: This is a PDF file of an unedited manuscript that has been accepted for publication. As a service to our customers we are providing this early version of the manuscript. The manuscript will undergo copyediting, typesetting, and review of the resulting proof before it is published in its final citable form. Please note that during the production process errors may be discovered which could affect the content, and all legal disclaimers that apply to the journal pertain.

Financial Disclosure: All authors have completed and submitted the ICMJE form for disclosure of potential conflicts of interest. None of the authors have any financial interests to disclose.

Contributions to Authors in each of these areas: design of the study (WRF, SL, CKC); conduct of the study (SL, JC); analysis of the study (LC, SL); writing article (CKC, SL, WRF); technical support (IK, GB, SE, MLG); obtaining funding (WRF, LC, CKC)
} 


\section{Introduction}

Diabetic macular edema (DME) is the most common cause of moderate vision loss in patients with diabetic retinopathy (DR). ${ }^{1}$ Vision loss is a result of the leakage of blood constituents from microaneurysms into the surrounding intra-retinal space which leads to retinal thickening and macular edema with image distortion. ${ }^{2}$

Histologic findings of microaneurysms in DR include pericyte loss, endothelial proliferation and apoptosis, and remodeling of basement membranes. These microaneurysms are found in close association with focal areas of capillary non-perfusion with a majority in the inner nuclear layer but also occasionally outer plexiform layer seen by electron microscopy. ${ }^{3}$

Since the Early Treatment Diabetic Retinopathy Study (ETDRS) for diabetic macular edema, focal/grid laser photocoagulation has been the mainstay of treating $\mathrm{DME}^{4,5}$ with fluorescein angiography (FA) guidance. More recently, the additional therapy with intravitreal anti-vascular endothelial growth factor (VEGF) antibody has increased treatment efficacy. ${ }^{6}$

Focal laser photocoagulation is thought to close microaneurysms directly which subsequently stops leakage of blood constituents into retinal space. Focal treatment is associated with reduction of macular edema in the occurrence of retinal thickening involving the center of macula. ${ }^{4}$ However, recurrence of DME is not uncommon and can be very difficult to treat despite multiple sessions of focal /grid laser photocoagulation. Difficulty in managing DME reflects our limited understanding of the pathophysiology of regressing microaneurysms following treatment. For decades, clinical evaluations of microaneurysms in DME were confined to fundus photography and FA. Even with the development of timedomain optical coherence tomography, individual details could not be appreciated.

More recently, visualization of diabetic microaneurysms has been characterized using spectral-domain optical coherence tomography (SD-OCT) ${ }^{7,8}$ Characterization of individual microaneurysms in DME following focal laser photocoagulation may increase our understanding of microaneurysm regression and provide insight to improve treatment approaches. In this study, we characterized individual microaneurysms in DME using simultaneous FA and SD-OCT following navigated focal laser photocoagulation.

\section{Methods}

\section{Study design}

We retrospectively assessed 123 microaneurysms by simultaneous FA and SD-OCT in 21 consecutive patients with nonproliferative proliferative diabetic retinopathy (NPDR) treated with navigated laser photocoagulation for DME at a single institution from November 2009 to January 2011. All treated microaneurysms which were visualized by FA and transected by a raster scan using SD-OCT were included in this study. Patients included 18 males and 3 females with an average age of $61.0 \pm 14.5$ years. A total of 29 eyes with mild (6/29), moderate (13/29), and severe (10/29) NPDR were followed at 3, 6, and 12 months following a single session of navigated laser photocoagulation performed within 7 days of baseline examination. At each visit, all patients underwent an ophthalmological examination with imaging studies including simultaneous FA and SD-OCT. Patients with proliferative diabetic retinopathy $(\mathrm{PDR})$, poorly controlled diabetes $\left(\mathrm{Hgb} \mathrm{A} 1_{\mathrm{C}}>8.0 \%\right)$, or poorly controlled hypertension (BP >140/90) were excluded. Eyes which did not undergo simultaneous FA and SD-OCT for all time points or received conventional laser photocoagulation were also excluded. Eyes which received any intravitreal steroid injection in the study eye or any intravitreal anti-VEGF in the study eye or contralateral eye within 6 
months prior to receiving navigated laser photocoagulation or during the follow up period were also excluded. This study adhered to the tenets of Declaration of Helsinki for research involving human subjects and was performed in compliance to the Institutional Review Board of the University of California, San Diego.

\section{Simultaneous fluorescein angiography and SD-OCT}

Simultaneous FA and SD-OCT images were acquired using the Spectralis HRA2 (Heidelberg Engineering, Carlsbad, CA) at each time points using the TruTrack ${ }^{\mathrm{TM}}$ Active Eye Tracking software of the device to co-localize angiographic findings with SD-OCT images. On FA, microaneurysm leakage was defined as a hyperfluorescent spot seen in early phase with increasing hyperfluorescence in late phase. ${ }^{9}$ Extent of microaneurysm leakage was graded as mild (score $=1$ ), moderate $($ score $=2$ ), or severe (score $=3$ ) leakage at each time point by two masked retina specialists. Closed microaneurysms by FA following laser photocoagulation were defined as an absence of focal hyperfluorescence in both early and late phases.

High resolution SD-OCT images were acquired using a raster scan mode $(20 \times 20$ degrees, 97 horizontal B-scans) during simultaneous FA imaging. Microaneurysms were detected as circular structures on SD-OCT which coincided with leakage on FA images. The follow-up scanning feature permitted reproducible SD-OCT image localization to characterize each microaneurysm over time. The follow-up feature has been validated by Spectralis HRA2 (Heidelberg Engineering, Carlsbad, CA) using retinal vessel landmarks to determine tracking information. SD-OCT images through the center of the microaneurysms were used to characterize leaking microaneurysms by two masked retina specialists.

Microaneurysm size was characterized by averaging the horizontal and vertical diameters of the outer and the inner wall (lumen) of each microaneurysm. Wall thickness was calculated as $:$ wall thickness $=($ outer diameter of microaneurysm - inner diameter of MA $) / 2$. All direct measurements were made using an internal caliper from the Spectralis HRA2. Central macular thickness within $500 \mu \mathrm{m}$ of the fovea and retinal thickness (RT) through the center of each microaneurysm were calculated from software in SD-OCT after localizing the center of fovea and the center of each MA. We determined the retinal layer in which the center of the microaneurysm was located. Reflectivity within the lumen of each microaneurysm was also determined to be heterogeneous or homogeneous based on the presence or absence of hyper-reflective spots on SD-OCT. Using SD-OCT, a closed microaneurysm following laser photocoagulation was defined as a microaneurysm with an absence or disappearance of a previously open lumen.

\section{Navigated focal laser photocoagulation}

To maximize treatment accuracy, leaking diabetic microaneurysms $(\mathrm{N}=123)$ were treated with focal laser photocoagulation $(532 \mathrm{~nm})$ using Navilas ${ }^{\circledR}$ (OD-OS, Teltow, Germany) within 7 days of examination at baseline. The navigated retinal laser photocoagulator has been used to increase accuracy to targeting microaneurysms and higher hit rate was reported. ${ }^{10}$ Multi-modality imaging (infrared, color, fluorescein angiography) integrated with a laser allowed planning and recording of treated areas. Eye tracking registration linked to the laser photocoagulator ensured accurate delivery of laser pulses in coordination with patient eye movements. In the present study, planning and treatment delivery of laser photocoagulation was performed by a single retina specialist who did not participate in the masked observations. Laser photocoagulation was performed with the following parameters: spot size $=100-130 \mu \mathrm{m}$, duration $=100 \mathrm{~ms}$, power $=70-150 \mathrm{~mW}$. Laser burn intensity was adjusted to achieve a gray-white burn by looking interchangeably at both real-time infrared and color images during treatment. 


\section{Statistical analysis}

Statistical analysis was performed using SAS statistical software (Version 9.2, SAS Inc, Cary, NC). Continuous variables were expressed as mean \pm standard deviation (SD). A paired $t$ test or $t$ test, and MacNemar's test or Chi-square test were used to compare continuous and categorical variables, respectively. Pearson's correlations were also performed among baseline characteristics. Since more than one microaneurysm were studied per eye, microaneurysm closure was used as a binary variable and analyzed using generalized estimation equations (GEE) to find significant predictors and to avoid any bias due to intrapatient or intra-eye correlations. Microaneurysm characteristics and microaneurysm closure by FA and SD-OCT were accessed by two masked retina specialists who did not perform laser photocoagulation. Agreement between two masked observers was assessed by calculating the concordant efficiency (Cohen's kappa coefficient). A $P$-value $<$. 05 was considered to be statistically significant.

\section{Results}

\section{Baseline characteristics of diabetic microaneurysms}

Leaking microaneurysms ( $\mathrm{N}=123)$ were characterized in 29 eyes (21 patients) with DME at baseline using simultaneous FA and SD-OCT (Table 1). Microaneurysms included those of mild (29/123, $23.6 \%)$, moderate (49/123, $39.8 \%)$ and severe (45/123, 36.6 \%) NPDR. All microaneurysms (123/123) were noted to have leakage with a moderate mean leakage score $(2.3 \pm 0.6)$ by FA. Using SD-OCT, mean central macular thickness within $500 \mu \mathrm{m}$ of the fovea was found to be $361 \pm 133 \mu \mathrm{m}$. Most of the studied microaneurysms were located in the inner nuclear layer (INL)/ inner plexiform layer (IPL) $(94 / 123,76.4 \%)$. A small number of microaneurysms were found in the retinal nerve fiber layer (RNFL)/ ganglion cell layer $(\mathrm{GCL})(11 / 123,8.9 \%)$ and outer nuclear layer $(\mathrm{ONL}) /$ outer plexiform layer $(\mathrm{OPL})(18 / 123$, $14.6 \%)$. Microaneurysm size was characterized by their outer and inner diameters, and wall thickness which resulted in $95 \pm 27 \mu \mathrm{m}, 59 \pm 21 \mu \mathrm{m}$, and $18 \pm 6 \mu \mathrm{m}$, respectively. Retinal thickness through the center of each microaneurysm averaged $422 \pm 89 \mu \mathrm{m}$. Using SDOCT, reflectivity within leaking microaneurysms was also characterized (Figure 1). Almost three-quarters of all microaneurysms $(91 / 123,74 \%)$ had a lumen with homogeneous reflectivity compared to one-quarter $(32 / 123,26 \%)$ with heterogeneous reflectivity.

As expected, increased FA leakage was associated with increased central macular thickness $(P<.05)$ and increased retinal thickness through the center of leaking microaneurysms $(P<$. $05)$ at baseline. Additionally, increased retinal thickness through the center of microaneurysms also correlated with increased central macular thickness $(P<.001)$. Interestingly, increased FA leakage was associated with increased microaneurysm outer and inner diameter $(P<.0001, P<.0001)$ as well increased wall thickness $(P<)$. Not surprisingly, increased outer diameters of microaneurysms also correlated with an increased inner diameter $(P<.0001)$ and increased wall thickness $(P<.0001)$.

\section{Closure rate of microaneurysms}

Microaneurysm closure following navigated laser photocoagulation at 3, 6, and 12 months was assessed using simultaneous FA and SD-OCT by two masked retina specialists (Table 2). The microaneurysm closure rate was increased at 6 and 12 months compared to 3 months by FA alone, SD-OCT alone, and both FA and SD-OCT. By FA alone, the microaneurysm closure rate was $72.4 \%(89 / 123), 83.7 \%(103 / 123)$, and $84.6 \%(104 / 123)$ at 3, 6, and 12 months, respectively. A significant increase in microaneurysm closure was observed at 6 and 12 months compared to 3 months $(P<.003, P<.002)$. However, no significant increase in microaneurysm closure was observed at 12 months compared to $6(P>.05)$. 
Similarly, evaluation by SD-OCT alone resulted in a microaneurysm closure rate of $70.0 \%$ (86/123), $80.5 \%$ (99/123), and $82.9 \%$ (102/123) at 3, 6, and 12 months, respectively. A significant increase in microaneurysm closure was also observed at 6 and 12 months compared to 3 months $(P<.004, P<.003)$ with no differnce in microaneurysm closure rate to 3 months compared to 6 months $(P>.05)$. Overall, there was no difference in microaneurysm closure rate between FA alone and SD-OCT alone at all time points $(P>$. 05).

Microaneurysm closure rate seen using both FA and SD-OCT similarly paralleled FA alone and SD-OCT alone. The microaneurysm closure rate seen by both FA and SD-OCT was $69.9 \%$ (84/123), $79.7 \%$ (98/123), and $82.9 \%$ (102/123) at 3, 6, and 12 months, respectively. A significant increase in microaneurysm closure by both FA and SD-OCT was observed at 6 and 12 months compared to 3 months $(P<.003, P<.001)$. No difference in microaneurysm closure at 12 months compared to 6 months was observed using FA and SDOCT together $(P>.05)$. Microaneurysm closure agreement between the two masked observers was $81.2 \%-83.2 \%($ Kappa $=0.85, P=.25)$

\section{Retinal thickness changes and closed microaneurysms characterized by SD-OCT}

Decreased central macular thickness and retinal thickness was seen following navigated focal laser photocoagulation at 12 months. Mean central macular thickness decreased by 49 $\pm 133 \mu \mathrm{m}(13.7 \%)$ from $361 \pm 133 \mu \mathrm{m}(225-638 \mu \mathrm{m})$ at baseline to $311 \pm 90 \mu \mathrm{m}(214-$ $604 \mu \mathrm{m})$ at 12 months after laser photocoagulation $(P<.0001)$. Retinal thickness also decreased by $71 \pm 82 \mu \mathrm{m}(16.8 \%)$ from $422 \pm 89 \mu \mathrm{m}(285-725 \mu \mathrm{m})$ at baseline to $352 \pm$ $78 \mu \mathrm{m}(242-605 \mu \mathrm{m})$ at 12 months following laser photocoagulation $(P<.0001)$.

Characterization of closed microaneurysms only using SD-OCT revealed that over half of the closed microaneurysms $(45 / 86,52.3 \%)$ left hyper-reflective spots in their original location while the remaining half $(41 / 86,47.7 \%)$ disappeared without any hyper-reflectivity on SD-OCT at 3 months (Figure 2, Figure 3). Consistent with baseline distribution of microaneurysms, a majority of hyper-reflective spots $(37 / 45,82.2 \%)$ were found in INL/ IPL and less frequently in ONL/OPL $(6 / 45,13.3 \%)$ and RNFL/GCL $(2 / 45,4.4 \%)$.

Similarly, a majority of closed microaneurysms which left no reflectivity were found in INL/IPL $(31 / 41,75.6 \%)$, while the remainder were found in ONL/OPL $(6 / 41,14.6 \%)$ and RNFL/GCL (4/41, $9.8 \%)$. Interestingly, microaneurysms which left hyper-reflective spots decreased at 6 months $(36 / 99,36.4 \%)$ and 12 months $(17 / 102,16.7 \%)$ compared to 3 months $(45 / 86,52.3 \%)(P<.003)$. A part of these hyper-reflective spots underwent complete loss of reflectivity at $6(16 / 45,35.6 \%)$ and 12 months $(21 / 36,58.3 \%)$.

Conversely, the absence of reflectivity was increased at 6 months $(63 / 99,63.6 \%)$ and 12 months $(85 / 102,83.3 \%)$ compared to 3 months $(41 / 86,47.7 \%)(P<.001)$.

A small number of microaneurysms which initially closed as hyper-reflective spots on SDOCT at 3 months were observed to reopen at the original location following navigated laser photocoagulation (3/45, 6.7 \%) (Figure 4). At 6 and 12 months, two and one microaneurysm respectively were observed to reopen by SD-OCT. All three of these microaneurysms which reopened were found to leak by FA. None of the closed microaneurysms which resulted in absence of hyper-reflectivity following laser photocoagulation subsequently re-opened or became hyper-reflective spots.

\section{Baseline characteristics associated with microaneurysm closure at 12 months}

Since microaneurysm closure stabilized between 6 and 12 months with minimal reopening of MAs, baseline characteristics associated with increased MA closure rate were analyzed by both FA and SD-OCT at 12 months. Baseline outer diameters of closed microaneurysms 
was $87 \pm 27 \mu \mathrm{m}$ while the outer diameter of unclosed microaneurysms $134 \pm 25 \mu \mathrm{m}$.

Baseline inner diameters of closed and unclosed microaneurysms were $52 \pm 21 \mu \mathrm{m}$ and $90 \pm$ $23 \mu \mathrm{m}$, respectively. Smaller outer and inner diameters at baseline were associated with an increased microaneurysm closure rate $(P<.0001, P<.001$, GEE).

Almost all microaneurysms with a heterogeneous lumen were closed (30/32, 93.8 \%) at 12 months. Microaneurysms with a homogeneous lumen at baseline resulted in a lower closure rate at 12 months following laser photocoagulation (72/91, 79.1\%). Microaneurysms which contained a heterogeneous lumen at baseline correlated with an increased microaneurysm closure rate at 12 months following laser photocoagulation $(P<.03$, GEE). Baseline outer and inner diameter of heterogeneous microaneurysms were $93.5 \pm 25.2 \mu \mathrm{m}$ and $57.1 \pm 23.6$ $\mu \mathrm{m}$, respectively. Baseline outer and inner diameter of homogeneous microaneurysms were $95.9 \pm 27.5 \mu \mathrm{m}$ and $59.5 \pm 20.3 \mu \mathrm{m}$, respectively. No difference in outer and inner diameter was observed between microaneurysms with a heterogeneous or homogeneous lumen $(P>$. $05, P>.05)$. No association was found between microaneurysm closure rate and patient characteristics (age, sex) or other baseline microaneurysm characteristics (grade of DR, FA leakage score, wall thickness, retinal thickness, and location).

\section{Discussion}

In this study, we characterized microaneurysm closure in diabetic macular edema following navigated focal laser photocoagulation using simultaneous FA and SD-OCT.

Microaneurysm closure rate following navigated laser determined by FA alone (89/123, 72.4 $\%)$, SD-OCT alone $(86 / 123,70.0 \%)$, and both FA and SD-OCT $(84 / 123,69.9 \%)$ at 3 months was similar to prior results using conventional laser. ${ }^{11}$ Interestingly, the microaneurysm closure rate in this study, continued to increase at 6 months compared to 3 months evaluated by FA alone, SD-OCT alone and both FA and SD-OCT $(P<.003, P<$. $004, P<.003)$ and stabilized at 12 months across all methods of evaluation. Additionally, both central macular thickness and retinal thickness at the location of microaneurysms were significantly decreased compared to baseline following navigated focal laser photocoagulation $(P<.0001, P<.0001)$. Our finding of delayed microaneurysm closure is consistent with decreased central subfield thickness reported as late as 8 months following a single treatment of focal laser photocoagulation by the diabetic retinopathy clinical research (DRCR) network. ${ }^{12}$

Baseline tomographical characteristics of leaking microaneurysms (outer and inner diameter, wall thickness, retinal thickness) in this study were similar to those previously reported. ${ }^{7}$ In patients with both NPDR and PDR, microaneurysm size did not correlate with FA leakage in the prior study. However, in this study, increased microaneurysm size (outer and inner diameter) and wall thickness correlated with increased FA leakage $(\mathrm{P}<.0001)$ when microaneurysms in patients with NPDR were evaluated. This suggests that MA size may have a greater influence on endothelial dysfunction in NPDR compared to PDR while other factors may contribute more predominantly in PDR.

Leaking microaneurysms with a heterogeneous lumen were associated with an increased closure rate following navigated laser photocoagulation $(P<.03)$. Prior studies have suggested that the hyper-reflective spots seen with SD-OCT in heterogeneous microaneurysm lumens are consistent with cellular components ${ }^{8}$ such as erythrocytes, leukocytes, and lipid deposits seen by electron microscopy. ${ }^{13}$ Increased hemoglobin containing cellular components increases laser absorption ${ }^{14-16}$ and may subsequently increase the rate of microaneurysm closure in those with a heterogeneous lumen. In addition, smaller baseline outer and inner diameters of microaneurysms were associated with increased closure rate at 12 months $(P<.0001, P<.001)$. It is not surprising that smaller 
microaneurysms with a higher heat absorption to surface area ratio may be more amenable to closure following laser photocoagulation.

Furthermore, leaking microaneurysms which initially closed at 3 months following laser photocoagulation resulted in either hyper-reflective spots $(45 / 86,52.3 \%)$ or absence of reflectivity $(41 / 86,47.7 \%)$ on SD-OCT. Interestingly, some of these hyper-reflective spots further underwent complete loss of reflectivity at 6 months $(16 / 45,35.6 \%)$ and 12 months $(21 / 36,58.3 \%)$. As the number of hyper-reflective spots decreased, the number of closed microaneurysms which resulted in complete loss of all reflectivity increased. Initial tomographical changes of a microaneurysm following laser photocoagulation, may be closure and collapse of its lumen to hyper-reflective spots with eventual resorption and disappearance of all reflectivity. Moreover, areas which lost all reflectivity never reaccumulated hyper-reflective spots. Complete loss of reflectivity observed by SD-OCT following focal laser may represent permanent microaneurysm closure with complete resorption of cellular components and lipid deposits. A small number of microaneurysms which were replaced with hyper-reflective spots were observed to re-open $(3 / 45,6.7 \%)$, while no re-opening was seen in areas of absence of residual reflectivity. Furthermore, reopen microaneurysms seen by SD-OCT was confirmed as leakage by FA. Although these numbers are small, hyper-reflective spots may represent collapsed microaneurysms which have the potential to re-open if there is no further cellular remodeling and resorption. A large prospective trial to monitor a greater number of microaneurysms following focal laser would provide a better understanding of the SD-OCT characteristics of microaneurysms prone to re-open and subsequently identify earlier need for re-treatment prior to the development of overt macular edema.

In summary, we have identified SD-OCT characteristics of microaneurysms which increase closure rate and resolution of DME following navigated laser photocoagulation.

Furthermore, we have characterized SD-OCT changes in microaneurysms during the course of microaneurysm closure following laser treatment as well as re-opening of a small number of initially closed microaneurysms. Detailed tomographical characteristics of closed microaneurysms following focal laser may improve our understanding of localized retinal changes which may lead to permanent microaneurysm closure, or may provide early detection of need for re-treatment.

\section{Acknowledgments}

Funding/Support: This study was supported by NIH R01EY007366 (WRF), NIH R01EY018589 (WRF), RPB incorporated and unrestricted funds from Jacobs Retina Center (WRF), NIH R01EY020617 (LC) and The Heed Ophthalmic Foundation (CKC).

Other Acknowledgements: none 


\section{Biographies}

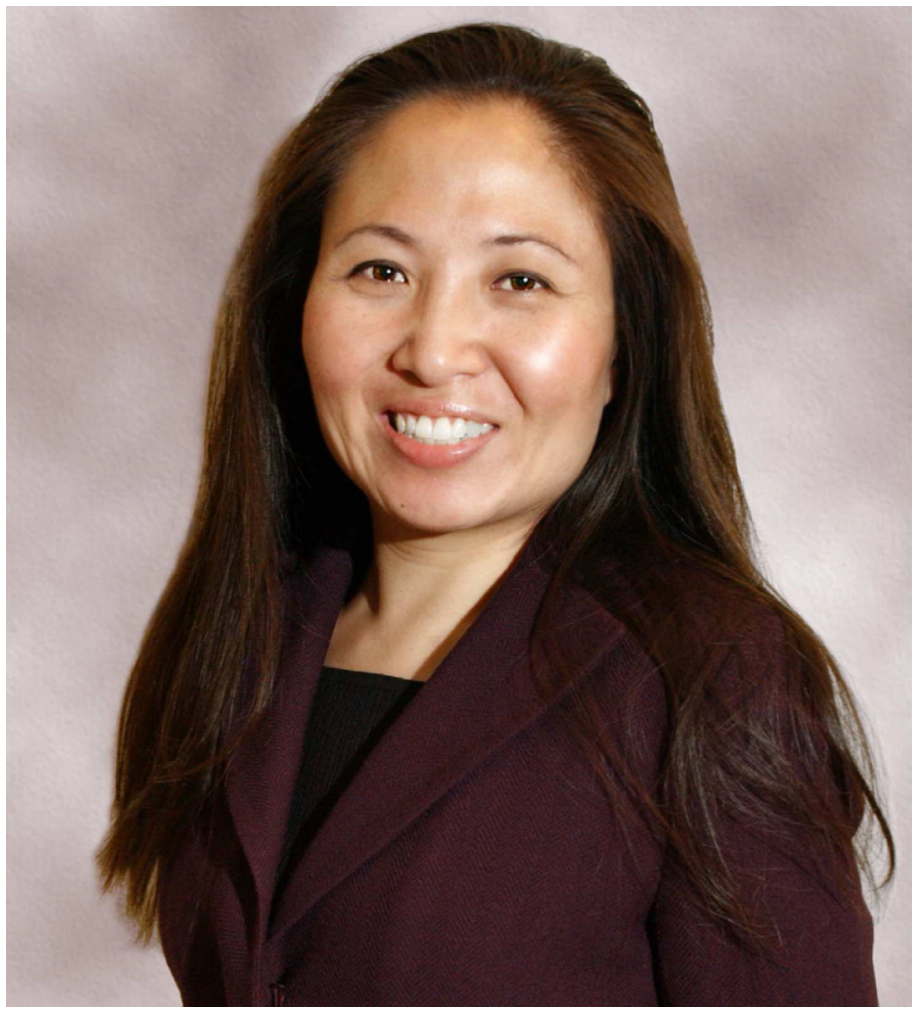

BIOSKETCH - SU NA LEE, MD, PhD

$\mathrm{Su} \mathrm{Na}$ Lee graduated from Chungnam University School of Medicine, Daejeon, Korea in 1998. She received her PhD degree from the Chungnam University Hospital, Daejeon, Korea and completed a vitreoretinal surgery fellowship at Seoul University Hospital, Seoul, Korea. Prior to her research fellowship at the University of California, San Diego, Dr. Lee was an associate professor at Eulji University Hospital, Daejeon, Korea. Dr. Lee is interested in diabetic macular edema and retinal angiogenesis. 


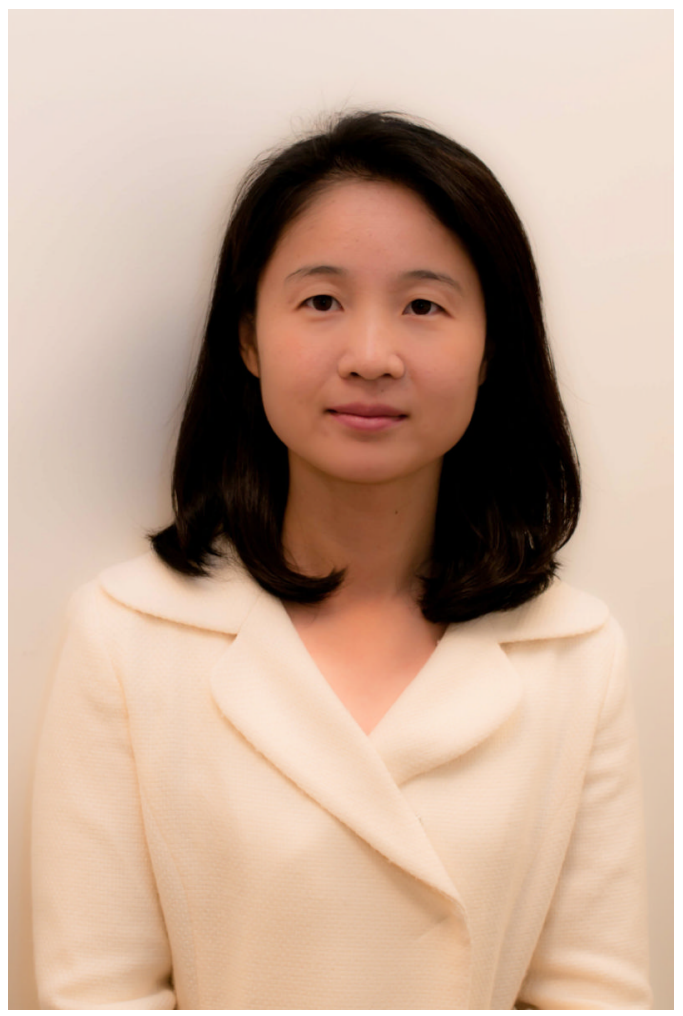

\section{References}

1. Moss SE, Klein R, Klein BE. The 14-year incidence of visual loss in a diabetic population. Ophthalmology. 1998; 105(6):998-1003. [PubMed: 9627648]

2. Murakami T, Nishijima K, Sakamoto A, Ota M, Horii T, Yoshimura N. Foveal cystoid spaces are associated with enlarged foveal avascular zone and microaneurysms in diabetic macular edema. Ophthalmology. 2011; 118(2):359-367. [PubMed: 20656355]

3. Stitt AW, Gardiner TA, Archer DB. Histological and ultrastructural investigation of retinal microaneurysm development in diabetic patients. Br J Ophthalmol. 1995; 79(4):362-367. [PubMed: 7742285]

4. Early Treatment Diabetic Retinopathy Study Research group. Photocoagulation for diabetic macular edema. Early Treatment Diabetic Retinopathy Study report number 1. Arch Ophthalmol. 1985; 103(12):1796-1806. [PubMed: 2866759]

5. Shah AM, Bressler NM, Jampol LM. Does laser still have a role in the management of retinal vascular and neovascular disease? Am J Ophthalmol. 2011; 152(3):332-339. [PubMed: 21742309]

6. Mitchell P, Bandello F, Schmidt-Erfurth U, et al. The RESTORE Study. Ranibizumab monotherapy or combined with laser versus laser monotherapy for diabetic macular edema. Ophthalmology. 2011; 118(4):615-625. [PubMed: 21459215]

7. Wang H, Chhablani J, Freeman WR, et al. Characterization of diabetic microaneurysms by simultaneous fluorescein angiography and spectral-domain optical coherence tomography. Am J Ophthalmol. 2012; 53(5):861-867. [PubMed: 22300473]

8. Horii T, Murakami T, Nishijima K, Sakamoto A, Ota M, Yoshimura N. Optical coherence tomographic characteristics of microaneurysms in diabetic retinopathty. Am J Ophthalmol. 2010; 150(6):840-848. [PubMed: 20855054]

9. Jalli PY, Hellstedt TJ, Immonen IJ. Early versus late staining of microaneurysms in fluorescein angiography. Retina. 1997; 17(3):211-215. [PubMed: 9196932] 
10. Kozak I, Oster SF, Cortes MA, et al. Clinical evaluation and treatment accuracy in diabetic macular edema using navigated laser photocoagulator NAVILAS. Ophthalmology. 2011; 118(6): 1119-1124. [PubMed: 21269701]

11. Gogi D, Gupta A, Gupta V, Pandav SS, Dogra MR. Retinal microaneurysmal closure following focal laser photocoagulation in diabetic macular edema. Ophthalmic Surg Lasers. 2002; 33(5): 362-367. [PubMed: 12358288]

12. Diabetic Retinopathy Clinical Research Network. The course of retina response to focal/grid photocoagulation for diabetic macular edema. Retina. 2009; 29(10):1436-1443. [PubMed: 19898182]

13. Bresnik GH, Davis MD, Myers FL, de Venecia G. Clinicopathologic correlations in diabetic retinopathy. II. Clinical and histologic appearances of retinal capillary microaneurysms. Arch Ophthalmol. 1977; 95(7):1215-1220. [PubMed: 880082]

14. Wallow IH, Bindley CD. Focal photocoagulation of diabetic macular edema. A clinicopathologic case report. Retina. 1988; 8(4):261-269. [PubMed: 3231918]

15. Trempe CL, Mainster MA, Pomerantzeff O, et al. Macular photocoagulation. Optimal wavelength selection. Ophthalmology. 1982; 89(7):721-728. [PubMed: 6181451]

16. Ulbig MW, McHugh DA, Hamilton AM. Diode laser photocoagulation for diabetic macular oedema. Br J Ophthalmol. 1995; 79(4):318-321. [PubMed: 7742274] 


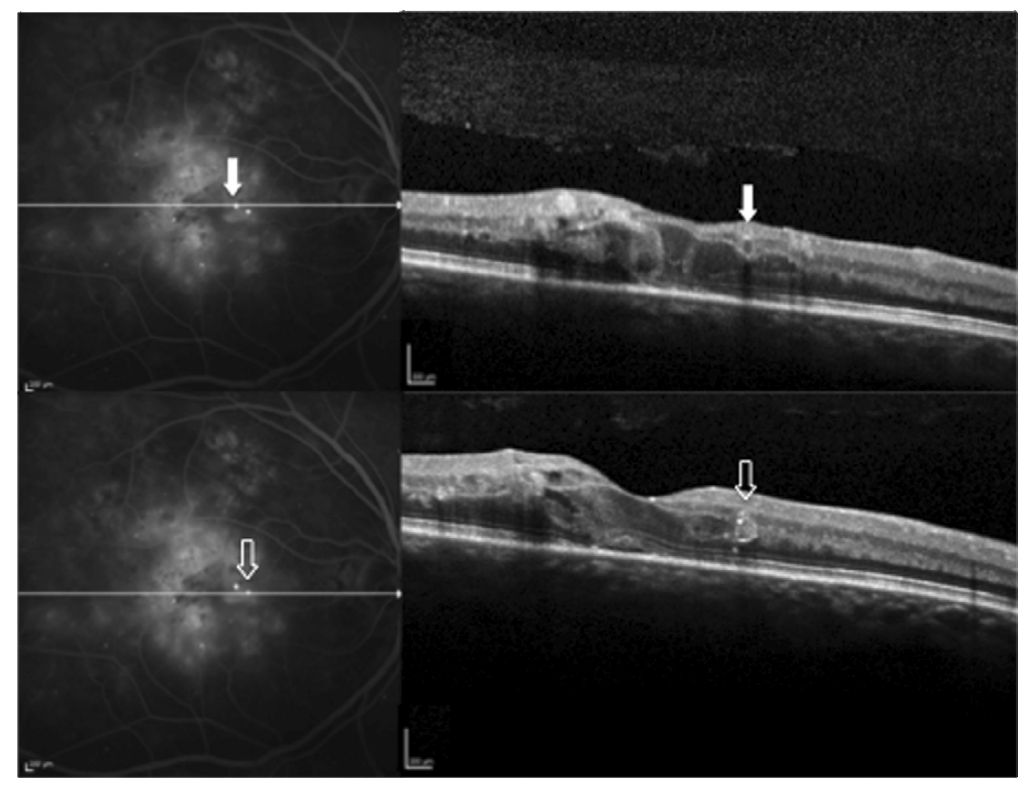

Figure 1.

Lumens of leaking retinal microaneurysms characterized by simultaneous fluorescein angiography (FA) and spectral-domain optical coherence tomography (SD-OCT) Representative simultaneous FA (left) and SD-OCT images (right) of leaking microaneurysms (white and open arrow) are seen in the inner nuclear layer with adjacent intra-retinal cystic edema. The microaneurysm lumen with homogeneous reflectivity (top right, white arrow) has uniform reflectivity unlike the microaneurysm lumen with heterogeneous reflectivity (bottom right, open arrow) which contains irregular hyperreflective spots. 


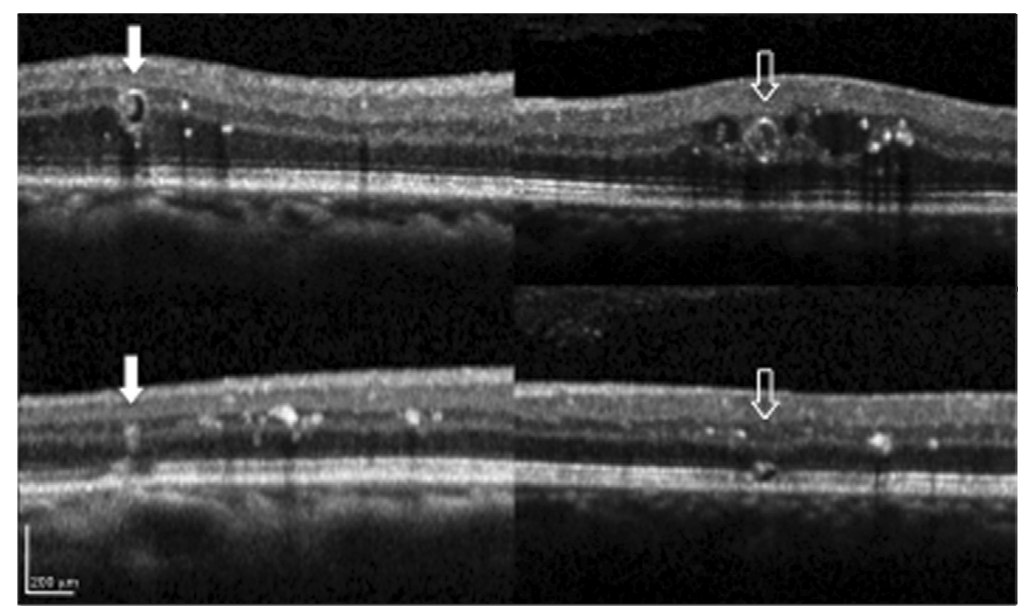

Figure 2.

Retinal microaneurysms characterized by spectral-domain optical coherence tomography following focal laser photocoagulation Leaking microaneurysms with a heterogeneous lumen (white and open arrows, top) in the inner nuclear layer with adjacent cystic edema were observed at baseline. At 6 months following navigated focal laser photocoagulation (bottom), a heterogeneous microaneurysm (white arrow) closed leaving hyper-reflective spots in its original location with resolution of adjacent cystic edema (bottom left). Another heterogeneous microaneurysm (open arrow) closed leaving an absence of any reflectivity following 6 months after laser photocoagulation (bottom right). 


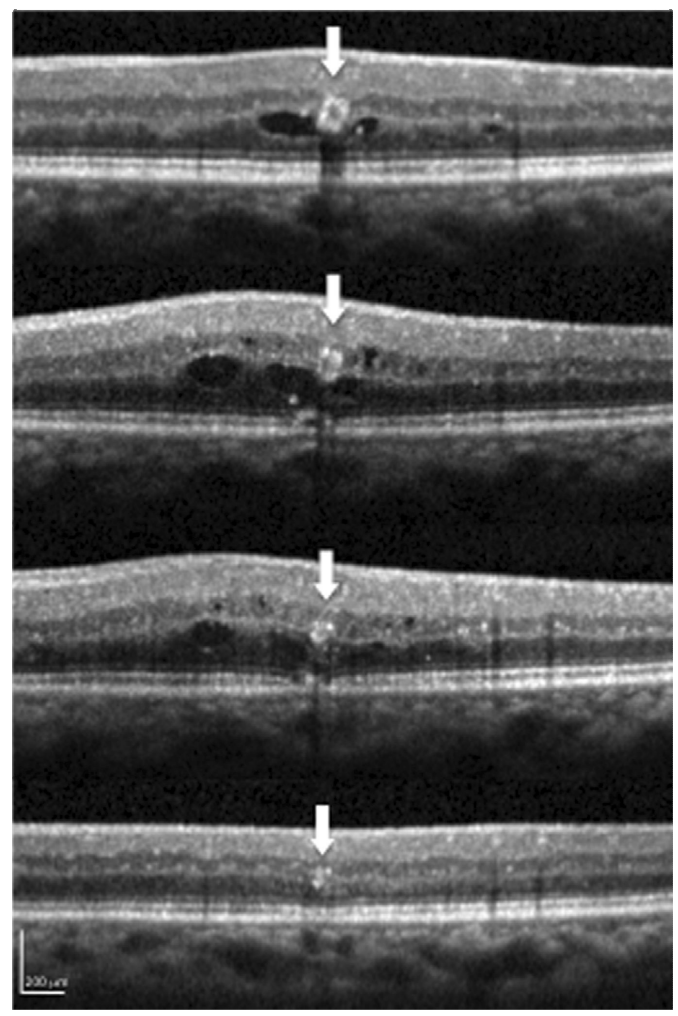

Figure 3.

Progression of retinal microaneurysm closure characterized by spectral-domain optical coherence tomography following focal laser photocoagulation

Microaneurysm with homogeneous lumen (white arrow) was observed in the inner nuclear layer with adjacent cystic fluid (top). At 3 months following focal laser photocoagulation, the microaneurysm has a persistently open lumen with cystic fluid (second row). At 6 months, the microaneurysm lumen has closed collapsing with decreased cystic fluid (third row). At 12 months (bottom), the lumen has been obliterated with leaving hyper-reflective spots in its original location with resolution of intra-retinal fluid. 


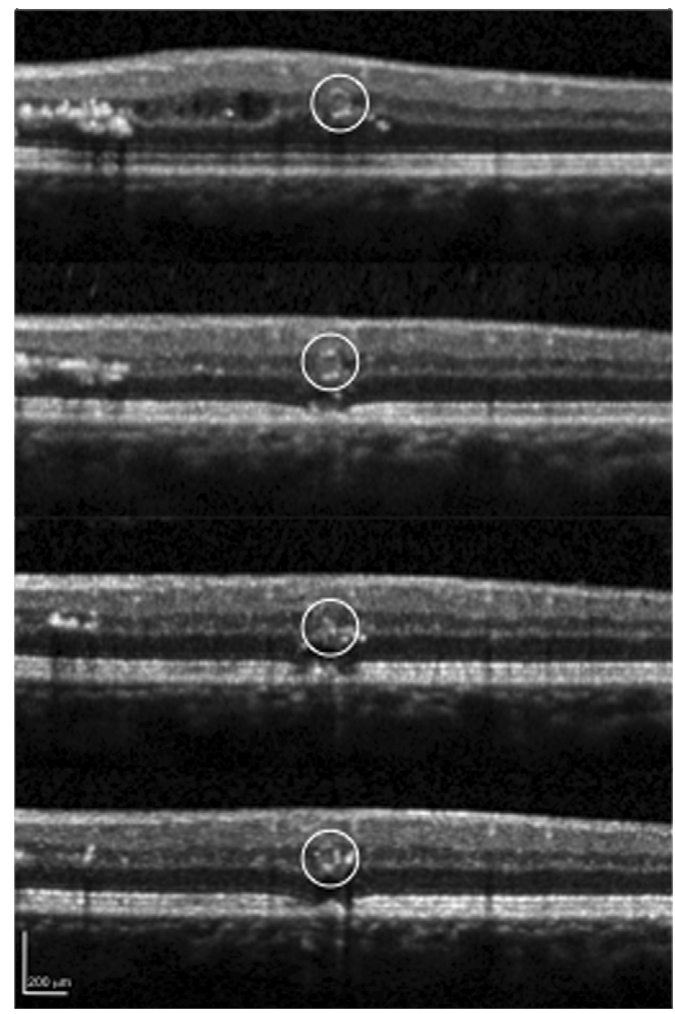

Figure 4.

Initially closed retinal microaneuryms may re-open following focal laser photocoagulation At baseline, a microaneurysm with a heterogeneous lumen (white circle) was observed in the inner nuclear layer with adjacent cystic fluid (top). At 3 months following focal laser photocoagulation, the microaneurysm has a persistently open lumen with decreased surrounding intra-retinal cystic fluid (second row). Disruption of the outer retina and retinal pigment epithelium by laser photocoagulation is also seen. At 6 months, the microaneurysm lumen has collapsed leaving hyper-reflective spots in its original location with resolution of adjacent intra-retinal fluid (third row). At 12 months, the microaneurysm has re-opened in the same location where hyper-reflective spots were seen previously (bottom). 
Table 1

Baseline characteristics of retinal microaneurysms

\begin{tabular}{lc}
\hline Baseline characteristic & Mean $(\mu \mathbf{m}) \pm$ SD (range) \\
\hline FA leakage score & $2.3 \pm 0.6(1-3)$ \\
Outer diameter & $95 \pm 27(60-186)$ \\
Inner diameter & $59 \pm 21(25-152)$ \\
Wall thickness & $18 \pm 6(5-36)$ \\
Retinal thickness & $422 \pm 89(285-725)$ \\
\hline Retinal layer & N/123 $(\%)$ \\
RNFL/GCL & $11(8.9)$ \\
INL/IPL & $94(76.4)$ \\
ONL/OPL & $18(14.6)$ \\
\hline Lumen reflectivity & N/123 $\%)$ \\
Homogeneous & $91(74.0)$ \\
Heterogeneous & $32(26.0)$ \\
\hline Grade of DR & N/123 $\%)$ \\
Mild NPDR & $29(23.6)$ \\
Moderate NPDR & $49(39.8)$ \\
Severe NPDR & $45(36.6)$ \\
\hline
\end{tabular}

$\mathrm{SD}=$ standard deviation, FA = fluorescein angiography, RNFL/GCL = retinal nerve fiber layer/ganglion cell layer, INL/IPL = inner nuclear layer/ inner plexiform layer, $\mathrm{ONL} / \mathrm{OPL}=$ outer nuclear layer/outer plexiform layer, $\mathrm{DR}=$ diabetic retinopathy, NPDR $=$ nonproliferative diabetic retinopathy 


\section{Table 2}

Closure rate of retinal microaneurysms

\begin{tabular}{lccc}
\hline Assessment & $\begin{array}{c}\text { 3 months } \\
\text { N/123 (\%) }\end{array}$ & $\begin{array}{r}\text { 6 months } \\
\text { N/123 }(\%)\end{array}$ & $\begin{array}{l}\text { 12 months } \\
\text { N/123 (\%) }\end{array}$ \\
\hline FA Alone & $89(72.4)$ & $103(83.7)$ & $104(84.6)$ \\
SD-OCT Alone & $86(70.0)$ & $99(80.5)$ & $102(82.9)$ \\
Both FA and SD-OCT & $84(69.9)$ & $98(79.7)$ & $102(82.9)$ \\
\hline
\end{tabular}

$\mathrm{FA}=$ fluorescein angiography, SD-OCT $=$ spectral-domain optical coherence tomography MA closure rate was increased at 6 months $(P<.003, P$ $<.004, P<.003)$ and 12 months $(P<.002, P<.003, P<.001)$ compared to 3 months by FA alone, SD-OCT alone, and both FA and SD-OCT. 